\title{
Análise da confiabilidade e da correlação de dois índices de estimativa da maturação esquelética: índice carpal e índice vertebral
}

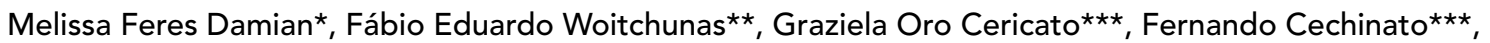

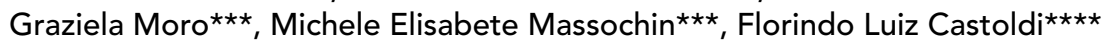

\section{Resumo}

Objetivo: o objetivo deste estudo foi avaliar a confiabilidade e a correlação de dois índices de estimativa da maturação esquelética. Metodologia: foi utilizada uma amostra de 210 radiografias carpais e telerradiografias laterais, de arquivo, de pacientes de ambos os gêneros, com idade entre 7 e 18 anos. As radiografias carpais foram utilizadas na determinação do Índice de Maturação Carpal (IMC) e as telerradiografias laterais na determinação do Índice de Maturação Vertebral (IMV). Cada grupo de radiografias foi examinado e reexaminado por 4 avaliadores, para analisar a confiabilidade de cada índice, e ainda foi realizada a comparação entre os estágios do IMC e do IMV, para avaliar a correlação entre os índices. Resultados: os resultados demonstraram que não houve diferença estatisticamente significante entre os 4 observadores nas avaliações do IMC e do IMV ( $p<0,00001)$, sendo as médias de correlação para o IMC de 95\% na primeira e 93,5\% na segunda avaliação, e para o IMV 84\% na primeira e $74 \%$ na segunda avaliação. Na correlação intra-avaliadores também não houve diferença estatisticamente significante para nenhum dos avaliadores $(\mathrm{p}<0,00001)$, onde a média para o IMC foi de $93,5 \%$ e para o IMV de $80 \%$. Na comparação entre os índices, mais uma vez não houve diferença estatisticamente significante $(\mathrm{p}<0,00001)$, sendo a correlação de $62 \%$ na primeira e de $80 \%$ na segunda avaliação. Conclusões: conclui-se que os dois índices mostraram-se confiáveis para estimar a maturação óssea e que há correlação entre os mesmos. Entretanto, sugere-se cautela na avaliação isolada pelo IMV.

Palavras-chave: Diagnóstico ortodôntico. Maturação esquelética. Radiografia carpal. Telerradiografia lateral. Vértebras cervicais.

\footnotetext{
* Doutora em Radiologia Odontológica pela FOP/Unicamp. Professora de Radiologia e Semiologia da Faculdade de Odontologia, Universidade de Passo Fundo.

** Mestre em Ortodontia pela Universidade Metodista de São Bernardo do Campo. Professor de Ortodontia da Faculdade de Odontologia, Universidade de Passo Fundo.

*** Cirurgiões-dentistas formados no curso de Odontologia da Universidade de Passo Fundo.

**** Estaticista da Universidade de Passo Fundo.
} 


\section{INTRODUÇÃO}

Diante de um caso de má oclusão, o profissional da área da Ortodontia/Ortopedia precisa reconhecer, entre outras coisas, se a desarmonia está sendo causada por uma discrepância dentária, esquelética ou dentoesquelética, para poder realizar o planejamento do melhor tratamento. Esta conduta deve-se ao fato de que as desarmonias de ordem dentária podem ser solucionadas em qualquer etapa da vida ${ }^{7,23,24}$, no entanto, as discrepâncias de ordem esquelética e dentoesqueléticas devem ser preferencialmente tratadas durante o período de resposta do tecido ósseo, ou seja, durante seu crescimento e maturação, pois, caso contrário, o tratamento proposto poderá não ser efetivo $^{1,2,5,7,12,13,17,19,21,23,24,25}$.

O crescimento e a maturação ósseos ou esqueléticos acompanham a tendência do crescimento geral do corpo, ocorrendo mais marcadamente durante a infância e a puberdade, e com uma velocidade decrescente, salvo pelos períodos de aceleração deste crescimento ou maturação chamados de surtos de crescimento ${ }^{28}$. É possivel reconhecer três surtos de aceleração na velocidade de crescimento: o primeiro ocorre na primeira infância, o segundo ocorre na segunda infância e, por fim, o terceiro, que coincide com a época da puberdade e por isso é chamado de Surto de Crescimento Puberal (SCP) ${ }^{7,28}$. Em função da precocidade dos pacientes durante a ocorrência do primeiro, principalmente, mas também do segundo surto de crescimento, o terceiro ou puberal é o período de maior aproveitamento para o tratamento ortopédico de discrepâncias ósseas $^{2,5,7,9,17,19,24,28 \text {. }}$

Ressalta-se, no entanto, que apesar do crescimento apresentar-se como um fenômeno constante e do SCP ocorrer, de um modo geral, em toda população saudável, estes fenômenos não acontecem na mesma idade cronológica para diferentes populações, ou mesmo para indivíduos dentro da mesma população $\mathrm{O}^{1,4,9,19,22,23,25,29}$. Isto porque este evento pode ser influenciado por fatores genéti- cos e raciais, condições climáticas, circunstâncias nutricionais, condições sócio-econômicas e alterações de uma maturação cada vez mais precoce do homem através do tempo ${ }^{19}$. Assim, a melhor maneira de se observar o crescimento e a maturação esquelética são as diferenciações em forma e tamanho apresentadas pelos ossos e que podem ser vistas radiograficamente ${ }^{7}$.

Várias áreas do corpo podem ser radiografadas e utilizadas para avaliar a maturação óssea, mas as radiografias de mão e punho (carpais) apresentam-se como as mais usadas neste propósito ${ }^{1,2,4,5,7-}$ 12,14-17,19,21,23,25,28,29. Ranke (1896 apud GUZZI, CARVALHO $^{15}$, 2000) e Rowland (1896 apud SANTOS, ALMEIDA ${ }^{23}$, 1999) foram os primeiros pesquisadores a analisarem o progresso do desenvolvimento ósseo por intermédio de radiografias de mão e punho. Desde então, vários métodos de avaliação deste tipo de radiografia foram criados, como o de Fishman ${ }^{9}$ e o de Grave e Brown ${ }^{14}$, chamados de Índices de Maturação Esqueléticos ou Carpais. A vasta utilização deste tipo de radiografia para o propósito de avaliar o crescimento e a maturação dos ossos deve-se ao fato de que possui um grande número de centros de ossificação em uma área relativamente pequena, pela facilidade da técnica radiográfica e pela pequena quantidade de radiação a que é exposto o paciente durante a realização do exame $e^{1,4,7,9,15,16,19,21,25}$.

Mas, mesmo que a avaliação de radiografias carpais possa ser um método testado e retificado na observação da maturação óssea ${ }^{10}$, alguns autores vêm pesquisando a utilização de outros métodos para realizar esta análise, a fim de facilitá-10 6,26 , de proporcionar uma alternativa para profissionais que não tem acesso à confecção de radiografias carpais ${ }^{24}$, mas principalmente de diminuir a dose de radiação a qual o paciente é exposto durante a realização de uma avaliação ortodôntica ${ }^{1-3,12,13,16-18,20-23,27}$. Ressalta-se o fato de que mesmo sendo a técnica carpal vantajosa, pela baixa dose de radiação exigida em sua confecção, constitui-se em uma exposição adicional, em um 
paciente que se encontra no período máximo de desenvolvimento ${ }^{21}$.

Neste sentido, Lamparski (apud ARAÚJO', 2001) publicou um estudo que avaliava as alterações morfológicas apresentadas durante $\mathrm{o}$ crescimento de vértebras cervicais, vistas em telerradiografias laterais, como método de análise da maturação esquelética. $\mathrm{O}$ autor justificou a escolha deste exame baseado no fato de que a telerradiografia lateral é utilizada rotineiramente nas avaliações ortodônticas e por isso diminuía a exposição dos pacientes à radiação ionizante. Desde então, diversos autores ${ }^{1-3,12,13,16,17,20-23,27}$ têm realizado pesquisas que buscam relacionar as alterações morfológicas das vértebras cervicais com o crescimento ósseo de indivíduos no período do SCP, sendo o método chamado de Índice de Maturação das Vértebras Cervicais ou, simplesmente, Índice de Maturação Vertebral. Entre estes autores estão Hassel e Farman ${ }^{16}$, que aprimoraram o método descrito por Lamparski (apud ARAÚ$\mathrm{JO}^{1}, 2001$ ) ao utilizarem na avaliação apenas as vértebras que poderiam ser observadas quando o paciente realiza uma telerradiografia lateral protegido da radiação ionizante por um avental plumbífero, que são as vértebras C2 (ou axis), C3 e C4. Ainda, destaca-se o trabalho de Baccetti et al. ${ }^{3}$, que criaram um novo método, baseado em cinco estágios de desenvolvimento, para avaliar o pico do desenvolvimento mandibular baseado nas alterações morfológicas e em medidas cefalométricas das vértebras cervicais $\mathrm{C} 2$ a $\mathrm{C} 4$.

No entanto, sabe-se que a utilização prática de um método de estimativa da maturação óssea só ocorre quando há plena confiança do profissional nos resultados obtidos por este ${ }^{8}$ e, também, que ainda não existem dados suficientes que mostrem a efetividade plena do método das vértebras cervicais para justificar a substituição do método carpal. A isto adiciona-se o fato de que populações diferentes podem responder de maneira distinta a um mesmo método de avaliação de maturação esquelética, pois crescem de maneira diferente $e^{1,4,25,28}$.
Neste contexto, objetivou-se com este estudo avaliar a confiabilidade do Índice de Maturação Esquelética Carpal (IMC) e do Índice de Maturação Esquelética Vertebral (IMV) na avaliação da maturação óssea, quando aplicados a uma população específica, por meio da reprodutibilidade dos métodos. Ainda, teve-se o propósito de analisar a possível correlação entre estes dois índices de maturação esquelética, para verificar a possibilidade do IMV ser usado de forma isolada para avaliar os estágios de maturação esquelética, diminuindo a dose de radiação por eliminar a radiografia carpal.

\section{MATERIAL E MÉTODOS Material}

A amostra desta pesquisa foi composta por um grupo de 210 radiografias, sendo 105 radiografias carpais (Fig. 1) e 105 telerradiografias laterais (Fig. 2) dos prontuários odontológicos da disciplina de Ortodontia e do curso de especialização em Ortodontia de uma instituição de ensino superior. Estas radiografias foram realizadas em pacientes que procuraram tratamento ortodôntico nesta instituição, provenientes das cidades da região de abrangência da universidade, entre os anos de 1998 e 2003.

\section{Métodos}

\section{Seleção da amostra}

Foram selecionados para a pesquisa apenas os prontuários que apresentavam pelo menos 1 radiografia carpal e 1 telerradiografia lateral com a mesma data de realização. Dentro desta seleção, foram excluídos os prontuários que apresentavam radiografias insatisfatórias para diagnóstico (com relação à técnica e ao processamento) e onde não era possivel visualizar por completo as vértebras cervicais $\mathrm{C} 2$, $\mathrm{C} 3$ e C4; bem como radiografias de pacientes com idade inferior a 7 e superior a 18 anos, uma vez que nestas idades o surto de crescimento puberal ainda não começou e já alcançou seu final, respectivamente, segundo outros trabalhos anteriormente realizados sobre o tema. 


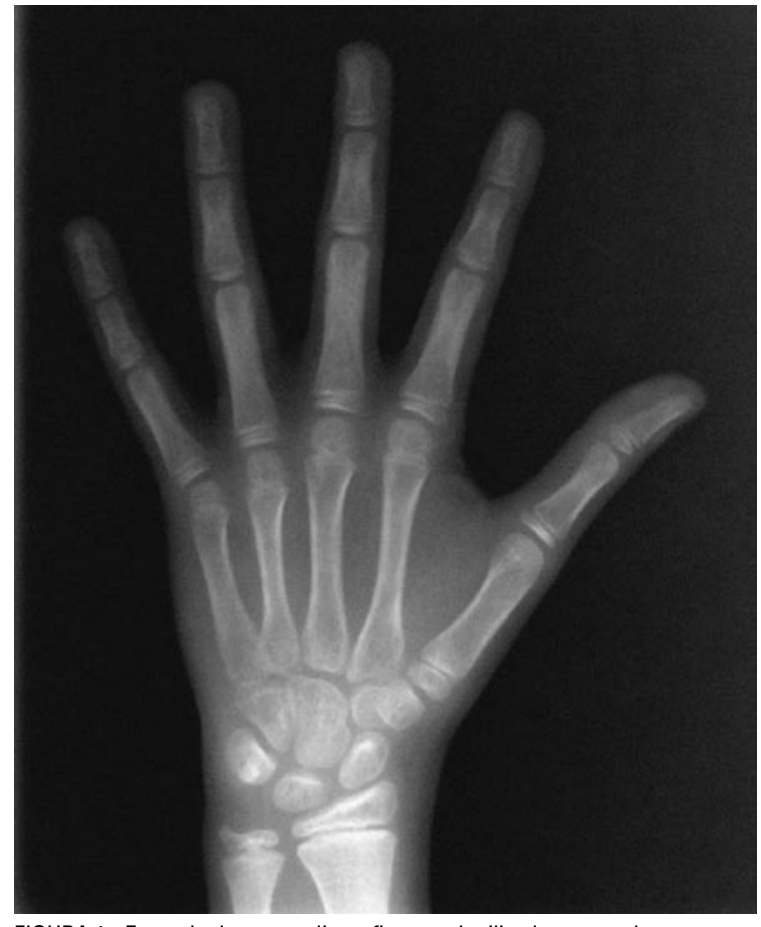

FIGURA 1 - Exemplo de uma radiografia carpal utilizada no estudo. Fonte: Disciplina de Ortodontia: FOUPF.

Também foram excluídas radiografias de pacientes onde houve a constatação de distúrbios metabólicos ou endócrinos que poderiam vir a influenciar o desenvolvimento normal.

Assim, de um total de 389 prontuários, foram selecionados 105 que apresentavam radiografias carpais e telerradiografias laterais com a mesma data de realização, de pacientes de ambos os gêneros, com idade entre 7 e 18 anos. Vale ressaltar que o intuito da pesquisa foi verificar a confiabilidade de cada índice de maturação esquelética e se havia correlação entre estes índices, não havendo pretensão de estimar o período do surto de crescimento puberal para esta população. Por isso, não houve segregação entre pacientes dos gêneros masculino e feminino.

\section{Avaliação das radiografias carpais}

Para a avaliação dos estágios de maturação observados nas radiografias carpais, foi utilizado o Índice de Maturação Carpal (IMC) proposto no

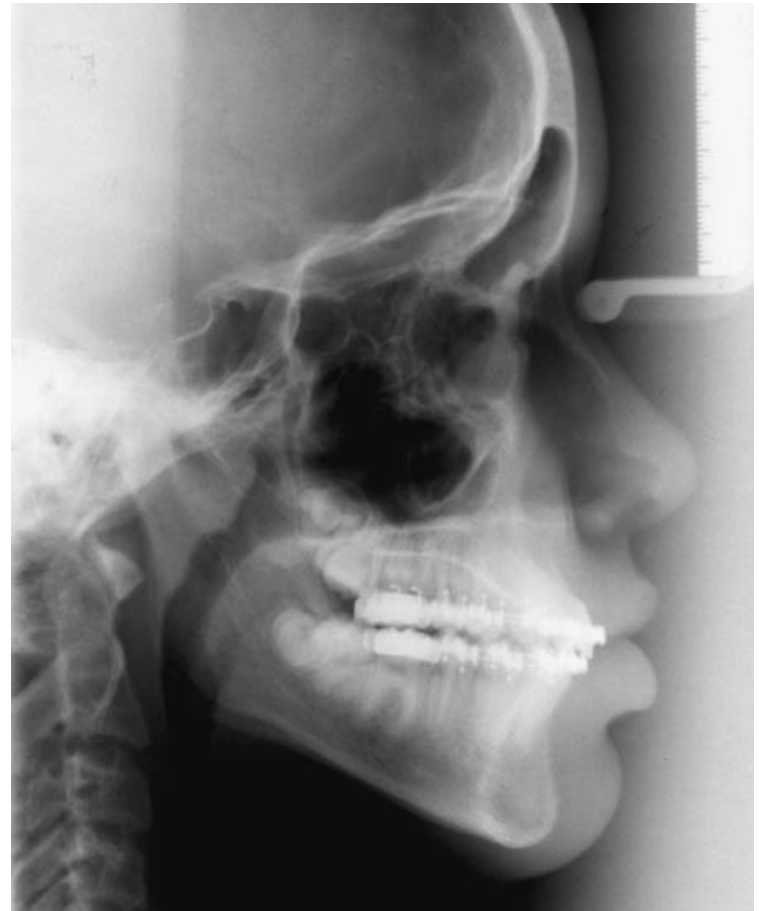

FIGURA 2 - Exemplo de uma telerradiografia lateral utilizada no estudo. Fonte: Disciplina de Ortodontia: FOUPF.

trabalho de Grave e Brown ${ }^{14}$. Este método avalia 14 eventos de calcificação na mão e no punho em relação à curva de velocidade de crescimento puberal (Quadro 1, Fig. 3).

\section{Avaliação das telerradiografias laterais}

Para análise do Índice de Maturação Vertebral (IMV), foi utilizado o método proposto por Hassel e Farman ${ }^{16}$, modificado do trabalho de Lamparski (apud ARAÚJO'1, 2001), que usa a telerradiografia lateral para classificar as vértebras cervicais C2 ou axis, $\mathrm{C} 3$ e C4, visualmente, de acordo com a morfologia do corpo de C3 e C4 e a formação de concavidade na borda inferior do processo odontóide do axis (C2), de $\mathrm{C} 3$ e $\mathrm{C} 4$, dentro das fases de maturação e do surto de crescimento puberal (Quadro 2, Fig. 4).

\section{Métodos de avaliação}

Cada um dos prontuários recebeu uma numeração seqüencial de 1 a 105, sendo que cada 


\begin{tabular}{|c|c|}
\hline estágio & descrição \\
\hline 1) $\mathrm{FP}_{2}$ & $\begin{array}{l}\text { epífise apresenta a mesma largura da diáfise na } \\
\text { falange proximal do segundo dedo }\end{array}$ \\
\hline 2) $\mathrm{FM}_{3}$ & $\begin{array}{l}\text { epífise apresenta a mesma largura da diáfise na } \\
\text { falange média do terceiro dedo }\end{array}$ \\
\hline 3) G-1 & $\begin{array}{l}\text { início da calcificação do gancho radiopaco do osso } \\
\text { hamato }\end{array}$ \\
\hline 4) Pisi & aparecimento do osso pisiforme \\
\hline 5) $R$ & $\begin{array}{l}\text { epífise apresenta a mesma largura da diáfise na epífise } \\
\text { distal do osso radio }\end{array}$ \\
\hline 6) $S$ & $\begin{array}{l}\text { início da calcificação do sesamóide ulnar na articula- } \\
\text { ção metacarpofalangeana do primeiro dedo }\end{array}$ \\
\hline 7) G-2 & calcificação total do gancho radiopaco do osso hamato \\
\hline 8) FM3cap & $\begin{array}{c}\text { capeamento epifisário na falange média do terceiro } \\
\text { dedo }\end{array}$ \\
\hline 9) FP1cap & $\begin{array}{l}\text { capeamento epifisário na falange proximal do primeiro } \\
\text { dedo }\end{array}$ \\
\hline 10) Rcap & capeamento epifisário na epífise distal do osso radio \\
\hline 11) FD3u & união epifisária na falange distal do terceiro dedo \\
\hline 12) FP3u & união epifisária na falange proximal do terceiro dedo \\
\hline 13) FM3u & união epifisária na falange média do terceiro dedo \\
\hline 14) R3u & união epifisária na epífise distal do osso radio \\
\hline
\end{tabular}

uma das radiografias (carpal e telerradiografia lateral) recebeu a mesma numeração do prontuário a que pertencia, para que pudessem ser avaliadas. Os dados como nome, idade cronológica e gênero dos pacientes, além do número original do prontuário de atendimento na Ortodontia foram anotados em uma relação, a qual os observadores do estudo não tiveram acesso durante as avaliações. Ainda, para evitar que os observadores tomassem conhecimento da idade cronológica e do gênero dos pacientes, gerando resultados tendenciosos, estas informações foram escondidas por meio da colocação de uma tira de cartolina preta sobre a identificação das radiografias, fazendo que as mesmas fossem identificadas somente pela numeração seqüencial atribuída no estudo ${ }^{22,23}$.

A análise da pesquisa foi realizada em três etapas. $\mathrm{Na}$ primeira, as radiografias foram avaliadas por 4 observadores, um radiologista e três acadêmicos de Odontologia, devidamente instruídos e

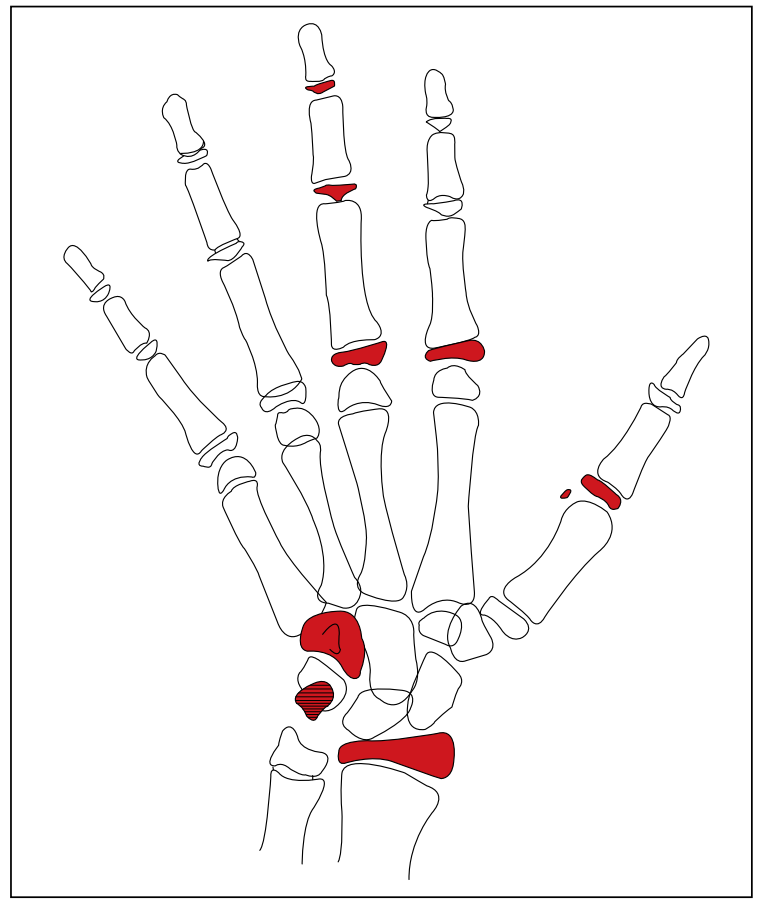

FIGURA 3 - Representação dos 14 estágios de maturação estudados por Grave e Brown ${ }^{14}$ para as radiografias carpais.

Fonte: adaptado de Grave, Brown ${ }^{14}$.

calibrados, denominados de $A_{1}, A_{2}, A_{3}$ e $A_{4}$, respectivamente, que classificaram cada radiografia dentro das fases de maturação do surto de crescimento puberal, por meio dos eventos de calcificação dos ossos da mão e do punho, para as radiografias carpais, e das vértebras cervicais, para as telerradiografias laterais. Passado 1 mês da primeira análise, esta foi repetida pelos mesmos 4 avaliadores (segunda etapa de análise). Esta metodologia serviu para a realização da correlação inter e intra-avaliadores, verificando a confiabilidade de cada índice, por meio da reprodutibilidade dos estágios atribuídos a cada radiografia por avaliadores diferentes e pelo mesmo avaliador em períodos diferentes.

Estas análises foram realizadas de forma isolada, ou seja, cada observador analisou cada tipo de radiografia separadamente, em dias distintos, e quando da realização das análises não houve comunicação entre os examinadores. Os dados relativos a cada observação foram anotados em fichas de ava- 
DAMIAN, M. F.; WOITCHUNAS, F. E.; CERICATO, G. 0.; CECHINATO, F.; MORO, G.; MASSOCHIN, M. E.; CASTOLDI, F. L.

\begin{tabular}{|c|c|}
\hline estágio & descrição \\
\hline 1) iniciação & $\begin{array}{c}\text { - bordos inferiores das vértebras C2, C3 e C4 são retos } \\
\text { - bordos superiores são afunilados, de posterior para anterior, dando ao corpo das vértebras C3 e C4 uma } \\
\text { forma trapezoidal (cunha) }\end{array}$ \\
\hline 2) aceleração & $\begin{array}{l}\text { - concavidades estão se desenvolvendo nos bordos inferiores das vértebras C2 e C3 } \\
\text { - bordo inferior da vértebra C4 está reto } \\
\text { - corpo das vértebras C3 e C4 está praticamente retangular }\end{array}$ \\
\hline 3) transição & $\begin{array}{l}\text { - concavidades distintas podem ser vistas nos bordos inferiores das vértebras C2 e C3 } \\
\text { - início do desenvolvimento de concavidade no bordo inferior da vértebra C4 } \\
\text { - corpo das vértebras C3 e C4 estão na forma retangular horizontal }\end{array}$ \\
\hline 4) desaceleração & $\begin{array}{l}\text { - concavidades distintas podem ser vistas nos bordos inferiores das vértebras C2, C3 e C4 } \\
\text { - corpo das vértebras C3 e C4 começa a ficar mais quadrado }\end{array}$ \\
\hline 5) maturação & $\begin{array}{l}\text { - concavidades mais acentuadas podem ser vistas nos bordos inferiores das vértebras C2, C3 e C4 } \\
\text { - corpo das vértebras C3 e C4 está na forma quadrangular }\end{array}$ \\
\hline 6) final & $\begin{array}{l}\text { - concavidades profundas presentes nos bordos inferiores das vértebras C2, C3 e C4 } \\
\text { - corpo de C3 e C4 estão quadrados ou com a dimensão vertical maior que a horizontal (retangular vertical) }\end{array}$ \\
\hline
\end{tabular}

QUADRO 2 - Estágios de maturação esquelética vertebrais descritos por Hassel e Farman ${ }^{16}$.

Fonte: Adaptado de Hassel, Farman ${ }^{16}$.

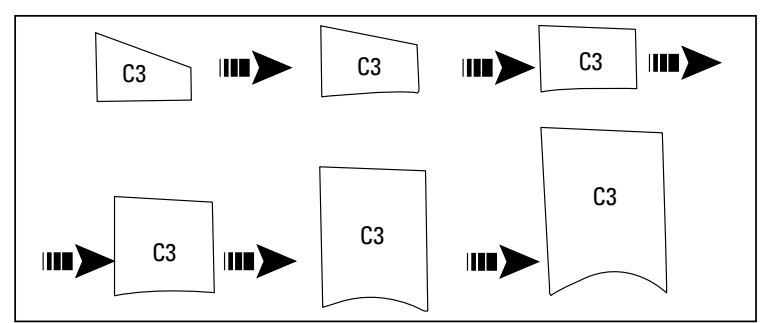

FIGURA 4 - Representação dos 6 estágios de maturação estudados por Hassel e Farman ${ }^{16}$ para as vértebras cervicais. Seqüência apresentada da esquerda para a direita e de cima para baixo: iniciação, aceleração, transição, desaceleração, maturação e final.

Fonte: Adaptado de Hassel e Farman ${ }^{16}$.

liação desenvolvidas para o estudo, sendo uma ficha para cada tipo de radiografia e para cada período de avaliação, para que o observador não tomasse conhecimento do estágio de maturação atribuído a um tipo de radiografia, quando estava avaliando outro e, também, para que não fosse influenciado por sua avaliação anterior para aquela radiografia.

Os resultados das avaliações das radiografias para cada índice de maturação esquelética (IMC e IMV) passaram por correlação inter-avaliadores, nos dois períodos de análise (inicial e após 1 mês), e correlação intra-avaliadores, para verificar o grau de confiabilidade de cada índice, por meio de um teste estatístico de correlação de Spearman para probabilidade de significância de $5 \%(\mathrm{p}<0,05)$.

Em uma terceira etapa, foram confrontados

\begin{tabular}{cc}
\hline IMC & IMV \\
\hline 1 e 2 & 1 \\
3,4 e 5 & 2 \\
6,7 e 8 & 3 \\
9 e 10 & 4 \\
11 & 5 \\
12,13 e 14 & 6 \\
\hline
\end{tabular}

QUADRO 3 - Comparação entre estágios de acordo com a curva de velocidade de crescimento puberal proposta por Grave, Brown ${ }^{14}$ e Hassel, Farman ${ }^{16}$.

os estágios de maturação obtidos em cada índice (IMC x IMV), para verificar se havia correlação entre os índices e, desta forma, a possibilidade da análise das vértebras cervicais ser usada de forma isolada para verificar o estágio de maturação do paciente, diminuindo a dose de radiação por eliminar a radiografia carpal. Esta avaliação também foi realizada pelo teste estatístico de correlação de Spearman para uma probabilidade de significância de 5\% ( $p<0,05)$. Em função da análise estatística, os 14 estágios do IMC foram agrupados em 6, coincidindo com os 6 estágios do IMV (Quadro 3), de acordo com a descrição dos autores ${ }^{14,16}$ para a posição de cada estágio dentro da curva que representava a velocidade de crescimento durante o SCP. 


\section{RESULTADOS}

As tabelas 1 e 2 explanam o resultado estatístico da correlação inter-observadores para o Índice de Maturação Carpal (IMC), no primeiro e segundo períodos de análise, respectivamente. As tabelas 3 e 4 representam o resultado estatístico da mesma correlação para o Índice de Maturação Vertebral (IMV), também para os dois períodos de análise, na mesma seqüência, ou seja, a tabela 3 refere-se ao primeiro período de análise e a tabela 4 à análise realizada 1 mês após a primeira.

A análise destas tabelas mostrou que, na avaliação inter-observadores, não houve diferença estatisticamente significante entre os 4 avaliadores do estudo na análise do IMC e do IMV $(\mathrm{p}<0,001)$. A média de correlação apresentada para o IMC na primeira avaliação foi de 95\% (94 a 96\%) e na segunda avaliação foi de 93,5\% (91 a 96\%), considerada altamente significante. Para o IMV, a média de correlação da primeira avaliação foi de $84 \%$ (77 a $91 \%$ ) e na segunda avaliação foi de $74 \%$ (70 a

Tabela 1 - Correlação inter-avaliadores para o Índice de Maturação Carpal no primeiro período de avaliação.

\begin{tabular}{cccc}
\hline avaliadores & $\mathbf{n}^{\mathbf{0}}$ radiografias & valor de $\mathbf{r}$ & valor de $\mathbf{p}$ \\
\hline $\mathrm{A}_{1} \times \mathrm{A}_{2}$ & 105 & 0,9682 & $0,00001^{*}$ \\
$\mathrm{~A}_{1} \times \mathrm{A}_{3}$ & 105 & 0,9454 & $0,00001^{*}$ \\
$\mathrm{~A}_{1} \times \mathrm{A}_{4}$ & 105 & 0,9536 & $0,00001^{*}$ \\
$\mathrm{~A}_{2} \times \mathrm{A}_{3}$ & 105 & 0,9615 & $0,00001^{*}$ \\
$\mathrm{~A}_{2} \times \mathrm{A}_{4}$ & 105 & 0,9677 & $0,00001^{*}$ \\
$\mathrm{~A}_{3} \mathrm{XA}_{4}$ & 105 & 0,9496 & $0,00001^{*}$ \\
\hline
\end{tabular}

* Estatisticamente significante para $p<0,05$

Tabela 3 - Correlação inter-avaliadores para o Índice de Maturação Vertebral no primeiro período de avaliação.

\begin{tabular}{cccc}
\hline avaliadores & $\mathbf{n}^{\mathbf{0}}$ radiografias & valor de $\mathbf{r}$ & valor de $\mathbf{p}$ \\
\hline $\mathrm{A}_{1} \times \mathrm{A}_{2}$ & 105 & 0,7857 & $0,00001^{*}$ \\
$\mathrm{~A}_{1} \times \mathrm{A}_{3}$ & 105 & 0,8307 & $0,00001^{*}$ \\
$\mathrm{~A}_{1} \times \mathrm{A}_{4}$ & 105 & 0,7845 & $0,00001^{*}$ \\
$\mathrm{~A}_{2} \times \mathrm{A}_{3}$ & 105 & 0,9103 & $0,00001^{*}$ \\
$\mathrm{~A}_{2} \times \mathrm{A}_{4}$ & 105 & 0,7753 & $0,00001^{*}$ \\
$\mathrm{~A}_{3} \times \mathrm{A}_{4}$ & 105 & 0,7796 & $0,00001^{*}$ \\
\hline
\end{tabular}

* Estatisticamente significante para $p<0,05$.
78\%), considerada significante, embora mais fraca em comparação à carpal.

As tabelas 5 e 6 apresentam os resultados obtidos na correlação intra-avaliadores para o IMC e para o IMV, respectivamente. Neste teste, a média da correlação para o IMC foi de 93,5\% (92 a 95\%) e para o IMV de $80 \%$ (74 a 86\%).

O que pode ser observado é que tanto na avaliação inter-observadores como na avaliação intraobservadores, as maiores médias de correlação foram alcançadas pelo IMC, ou seja, pela utilização da radiografia carpal. Entretanto, as médias apresentadas pelo IMV também foram consideradas estatisticamente significantes.

O resultado da correlação entre os índices estudados (IMC X IMV) está apresentado na tabela 7. Nesta correlação mais uma vez não houve diferença estatisticamente significante ( $\mathrm{p}<0,001)$, sendo a média de correlação $71 \%$ (62\% na primeira avaliação e $80 \%$ na segunda avaliação), considerada significante especialmente no segundo período de avaliação.

Tabela 2 - Correlação inter-avaliadores para o Índice de Maturação Carpal no segundo período de avaliação.

\begin{tabular}{cccc}
\hline avaliadores & $\mathbf{n}^{\mathbf{0}}$ radiografias & valor de $\mathbf{r}$ & valor de $\mathbf{p}$ \\
\hline $\mathrm{A}_{1} \times \mathrm{A}_{2}$ & 105 & 0,9178 & $0,00001^{*}$ \\
$\mathrm{~A}_{1} \times \mathrm{A}_{3}$ & 105 & 0,9259 & $0,00001^{*}$ \\
$\mathrm{~A}_{1} \times \mathrm{A}_{4}$ & 105 & 0,9323 & $0,00001^{*}$ \\
$\mathrm{~A}_{2} \times \mathrm{A}_{3}$ & 105 & 0,9454 & $0,00001^{*}$ \\
$\mathrm{~A}_{2} \times \mathrm{A}_{4}$ & 105 & 0,9505 & $0,00001^{*}$ \\
$\mathrm{~A}_{3} \times \mathrm{A}_{4}$ & 105 & 0,9606 & $0,00001^{*}$ \\
\hline
\end{tabular}

* Estatisticamente significante para $p<0,05$.

Tabela 4 - Correlação inter-avaliadores para o Índice de Maturação Vertebral no segundo período de avaliação.

\begin{tabular}{cccc}
\hline avaliadores & $\mathbf{n}^{0}$ radiografias & valor de $\mathbf{r}$ & valor de $\mathbf{p}$ \\
\hline $\mathrm{A}_{1} \times \mathrm{A}_{2}$ & 105 & 0,7832 & $0,00001^{*}$ \\
$\mathrm{~A}_{1} \times \mathrm{A}_{3}$ & 105 & 0,7039 & $0,00001^{*}$ \\
$\mathrm{~A}_{1} \times \mathrm{A}_{4}$ & 105 & 0,7030 & $0,00001^{*}$ \\
$\mathrm{~A}_{2} \times \mathrm{A}_{3}$ & 105 & 0,7835 & $0,00001^{*}$ \\
$\mathrm{~A}_{2} \times \mathrm{A}_{4}$ & 105 & 0,7333 & $0,00001^{*}$ \\
$\mathrm{~A}_{3} \times \mathrm{A}_{4}$ & 105 & 0,7734 & $0,00001^{*}$ \\
\hline
\end{tabular}

* Estatisticamente significante para $\mathrm{p}<0,05$. 
Tabela 5 - Correlação intra-avaliadores para o Índice de Maturação Carpal.

\begin{tabular}{cccc}
\hline avaliador & $\mathbf{n}^{\mathbf{0}}$ radiografias & valor de $\mathbf{r}$ & valor de $\mathbf{p}$ \\
\hline $\mathrm{A}_{1}$ & 105 & 0,9260 & $0,00001^{*}$ \\
$\mathrm{~A}_{2}$ & 105 & 0,9528 & $0,00001^{*}$ \\
$\mathrm{~A}_{3}$ & 105 & 0,9440 & $0,00001^{*}$ \\
$\mathrm{~A}_{4}$ & 105 & 0,9364 & $0,00001^{*}$ \\
\hline
\end{tabular}

* Estatisticamente significante para $p<0,05$.

Tabela 7 - Correlação entre o Índice de Maturação Carpal e o Índice de Maturação Vertebral.

\begin{tabular}{cccc}
\hline avaliação & $\mathbf{n}^{\mathbf{0}}$ radiografias & valor de $\mathbf{r}$ & valor de $\mathbf{p}$ \\
\hline $1^{\text {a }}$ & 105 & 0,6259 & $0,00001^{*}$ \\
$2^{\text {a }}$ & 105 & 0,8042 & $0,00001^{*}$ \\
\hline
\end{tabular}

* Estatisticamente significante para $p<0,05$.

\section{DISCUSSÃO}

Após consulta à literatura pertinente ao tema e com a pretensão de adicionar dados sobre a utilização do Índice de Maturação Vertebral (IMV), especialmente com relação ao seu grau de confiabilidade e de correlação com o Índice de Maturação Carpal (IMC), uma vez que ainda são escassos os trabalhos que tratam do assunto a ponto de sugerir que a avaliação da maturação esquelética por radiografias carpais possa ser substituída pelo método das vértebras cervicais, foi desenvolvido este estudo.

Para testar a confiabilidade, foi analisada a capacidade de reprodutibilidade das avaliações do IMV e do IMC por testes inter e intra-observadores. Foi possivel perceber que as maiores médias de correlação, nas duas avaliações, foram alcançadas pelo IMC, quando comparado ao IMV.

$\mathrm{Na}$ correlação inter-avaliadores, as médias para o IMC foram de 95 e 93,5\% respectivamente no primeiro e segundo período de avaliação, e para o IMV, de 84 a 74\%, na mesma ordem. Em trabalhos semelhantes, Araújo ${ }^{1}$ encontrou médias de correlação de $80 \%$ para o IMC e de 38\% para o IMV; Hassel e Farman ${ }^{16}$ chegaram a valores de $99 \%$ para o IMC e $87,5 \%$ para o IMV; Kucukkeles et al. ${ }^{17}$,
Tabela 6 - Correlação intra-avaliadores para o Índice de Maturação Vertebral.

\begin{tabular}{cccc}
\hline avaliador & $\mathbf{n}^{\mathbf{0}}$ radiografias & valor de $\mathbf{r}$ & valor de $\mathbf{p}$ \\
\hline $\mathrm{A}_{1}$ & 105 & 0,7465 & $0,00001^{*}$ \\
$\mathrm{~A}_{2}$ & 105 & 0,7824 & $0,00001^{*}$ \\
$\mathrm{~A}_{3}$ & 105 & 0,8665 & $0,00001^{*}$ \\
$\mathrm{~A}_{4}$ & 105 & 0,8221 & $0,00001^{*}$ \\
\hline
\end{tabular}

* Estatisticamente significante para $p<0,05$.

de $89 \%$ para o IMC e de $87,3 \%$ para o IMV e Santos e Almeida ${ }^{23}$, de $95 \%$ para o IMC e de $82 \%$ para o IMV. Já Santos et al. ${ }^{22}$, trabalhando apenas com avaliação do IMV em telerradiografias laterais, obtiveram como resultado para avaliação inter-observadores um valor médio de $82 \%$. Como pode ser constatado, os resultados deste estudo assemelham-se aos já divulgados na literatura, que também ressaltam valores de correlação inter-avaliadores maiores quando as radiografias carpais são utilizadas na avaliação da maturação óssea. Entretanto, destaca-se que os valores encontrados para o IMV na segunda fase de avaliação deste estudo (realizada 1 mês após a primeira) foram inferiores aos citados nos trabalhos anteriormente realizados sobre o tema, com metodologia semelhante à utilizada nesta pesquisa, com exceção de Araújo ${ }^{1}$, que encontrou um valor médio de correlação de 38\% para o IMV variando de 98 a 18\%, dependendo da faixa etária e do gênero dos pacientes analisados.

As médias de correlação intra-avaliadores obtidas neste estudo situaram-se em $93,5 \%$ para o IMC e $80 \%$ para o IMV, ressaltando mais uma vez a superioridade das médias do IMC em relação ao IMV. Araújo ${ }^{1}$ chegou a valores médios de correlação de $91 \%$ para o IMC e de $61 \%$ para o IMV; García-Fernandes et al. ${ }^{12}$, quando testaram a correlação intra-avaliadores do IMV, encontraram média de $90 \%$; Hassel e Farman ${ }^{16}$ obtiveram média de correlação intra-avaliadores de $100 \%$ tanto para o IMC como para o IMV; Kucukkeles et al. ${ }^{17}$ encontraram valores médios de correlação de $90 \%$ para o IMC e de $83 \%$ para o IMV; Santos e Almeida ${ }^{23}$, 
de $97 \%$ para o IMC e de $90 \%$ para o IMV e Santos et al..$^{22}$ encontraram correlação de $90 \%$ na avaliação intra-avaliadores para o IMV. Os resultados encontrados assemelham-se aos trabalhos de Araújo ${ }^{1}$, Kucukkeles et al. ${ }^{17}$ e de Santos e Almei$\mathrm{da}^{23}$, que também relataram médias de correlação maiores para o IMC, mas diferem da igualdade de médias encontradas por Hassel e Farman ${ }^{16}$. Os valores médios de correlação intra-avaliadores para o IMV, obtidos neste estudo, foram menores que os dos trabalhos encontrados na literatura e supracitados, salvo o trabalho de Araújoㅁ, que encontrou média de $61 \%$ de correlação, enquanto neste estudo foi encontrada média de $80 \%$, repetindo o resultado encontrado na avaliação inter-observadores.

Concordando com Fishman ${ }^{9}$, acredita-se que o método que utiliza o IMC mostrou maior reprodutibilidade e assimilação que o IMV, uma vez que as médias de correlação inter e intra-avaliadores foram maiores, em função do número maior de centros de ossificação que são utilizados nos diferentes métodos que avaliam a maturação esquelética por meio de radiografias carpais. Esta conclusão está baseada no fato de que mesmo utilizando uma metodologia diferente para avaliação do IMC (método de Grave e Brown ${ }^{14}$ em comparação com a maior parte dos trabalhos ${ }^{12,16,17,22,23}$ citados na literatura,que utilizaram o método de Fishman ${ }^{9}$ ), os resultados encontrados foram semelhantes aos destes autores. Ao contrário, acreditase que a hipótese defendida por Santos e Almei$\mathrm{da}^{23}$, sobre a superioridade do método carpal em função da familiarização dos examinadores com os eventos de ossificação da mão e do punho, não seja verdadeira, uma vez que 3 dos 4 avaliadores deste estudo não possuíam qualquer familiarização anterior com nenhum dos métodos. Ainda concorda-se com Generoso et al. ${ }^{13}$ que citam que uma das grandes dificuldades para a utilização do IMV pelo método de Hassel e Farman ${ }^{16}$ modificado de Lamparski (apud ARAÚJO'1 , 2001), que foi o método utilizado neste estudo, seria a falta de aplicabilidade dos aspectos anatômicos descritos no estudo, pois parece haver estágios intermediários aos 6 estágios citados pelos autores. Somado a isto não se pode deixar de relatar o trabalho de O’Reilly e Yanniello ${ }^{20}$, que citaram que a avaliação do IMV poderia ser prejudicada pela sutileza das alterações morfológicas que sofrem as vértebras durante o seu crescimento e também pela postura incorreta do paciente no momento da confecção da telerradiografia, que causaria sobreposição das estruturas e, desta forma, dificuldade na avaliação das mesmas.

Porém, mesmo que as médias de correlação tenham sido maiores para o IMC, não houve diferença estatisticamente significante nas avaliações do IMV, tanto inter como intra-observadores $(\mathrm{p}<0,00001)$, o que torna o método válido para avaliar a estimativa da maturação esquelética na população estudada. Este resultado concorda com achados anteriores ${ }^{1,2,12,13,16-17,20-23,27}$, que propõem a utilização do Índice de Maturação Vertebral para análise da maturidade óssea.

Assim como na avaliação inter-observadores, a correlação entre os dois índices (IMC e IMV) foi testada nos dois períodos de análise do estudo (inicial e após 1 mês). Nestes dois períodos não houve diferença estatisticamente significante entre os dois índices de maturação óssea estudados $(\mathrm{p}<0,00001)$, sendo o valor médio de correlação em torno de $71 \%$ (62\% na primeira e $80 \%$ na segunda avaliação). Estes resultados confirmam os anteriores, realizados para avaliar a confiabilidade dos métodos, com relação à validade do IMV para a análise da maturação esquelética. Outros autores encontraram correlações ao nível de $94 \%^{12}, 89 \%^{16}$, $74 \%{ }^{17}, 78 \%^{21}$ e $68 \%{ }^{23}$ na comparação entre os índices carpal e vertebral. No segundo período de avaliação, o resultado deste estudo para a correlação IMC X IMV foi inferior aos achados de Hassel e Farman ${ }^{16}$ e, principalmente, García-Fernandes et al. ${ }^{12}$, mas foi superior aos dos outros autores, mesmo que muito semelhante aos de San Román et al. ${ }^{21}$ Contudo, no primeiro período de avaliação, 
o resultado de $62 \%$ de correlação foi inferior aos aqui comparados, e ainda considerado estatisticamente baixo, mesmo que significante.

Uma possível explicação para este resultado está no fato de que, como anteriormente citado, somente 1 dos 4 avaliadores do estudo apresentava conhecimento e familiarização anterior com o assunto pesquisado e os métodos utilizados. Esta hipótese é reforçada quando a correlação entre índices no segundo período de avaliação alcançou um resultado de $80 \%$.

Desta forma, foi possivel concordar com outros autores ${ }^{1,2,10,17,20-23,27}$ e reforçar a idéia de que, mesmo sendo um método válido na análise da maturação óssea, o IMV, avaliado em telerradiografias laterais, deve ser usado como método auxiliar do IMC até que possa haver familiarização com este e, assim, maior confiabilidade na sua utilização. Isto porque, em concordância com Coutinho et al. ${ }^{8}$, um método de avaliação de maturidade somente será aplicável quando o profissional que utilizá-lo obtiver conhecimento e confiança nele.

\section{CONCLUSÕES}

Após a aplicação da metodologia, coleta e discussão dos resultados, pôde-se concluir que:

- tanto o Índice de Maturação Carpal (IMC) quanto o Índice de Maturação Vertebral (IMV), de acordo com os métodos de avaliação propostos neste estudo, são confiáveis para avaliar a maturação óssea na população delimitada para a pesquisa;

- houve correlação positiva entre os dois índices de maturação esquelética avaliados (IMC e IMV);

- sugere-se que o profissional tenha cautela em considerar o exame das vértebras cervicais um método absoluto para avaliação da maturação esquelética de pacientes em crescimento, enquanto não houver familiarização deste profissional com o método.

\title{
Reliability and correlation analysis of two skeletal maturation evaluation indexes: hand-wrist index and cervical vertebrae index
}

\begin{abstract}
Aim: the aim of this study were to evaluate the reliability and correlation of two different skeletal maturation evaluation indexes. Methods: two hundred and ten hand-wrist and cephalometric radiographs from files of patients with 7- 18 years old were used. The hand-wrist radiographs were used to determine the skeletal maturation index (SMI) and the cephalometric radiographs to determine the cervical vertebrae maturation index (CVMI). Four independent evaluators were asked to examine twice each radiography and to verify the reliability of each index. The stages of $\mathrm{SMI}$ and CVMI were compared to evaluate the relationship between them. Results: the results were not statisticaly different among the four evaluators on SMI and CVMI indexes $(p<0.00001)$. The correlation average to SMI was $95 \%$ for the first evaluation and $93.5 \%$ for the second evaluation. On the CVMI, the correlation average was $84 \%$ for the first evaluation and $74 \%$ for the second evaluation. The correlation intraoperator did not showed significant difference $(p<0.00001)$ and the average for SMI was $93.5 \%$ and for CVMI was $80 \%$. Comparing the indexes, there was no statistical differences $(p<0.00001)$ with correlation of $62 \%$ in the first evaluation and $80 \%$ in the second evaluation. Conclusion: results from the two indexes suggest they are reliable to estimate the skeletal maturation. There is a correlation between the two indexes, however caution must be taken when using only the CVMI index.
\end{abstract}

Key words: Orthodontic diagnosis. Skeletal maturation. Hand-wrist radiographs. Cephalometric radiographs. Cervical vertebrae. 


\section{REFERÊNCIAS}

1. ARAÚJO, T. S. S. Estudo comparativo entre dois métodos de estimativa da maturação óssea. 2001. Dissertação (Mestrado)-Faculdade de Odontologia de Piracicaba, Universidade Estadual de Campinas, Piracicaba, 2001.

2. ARMOND, M. C.: CASTILHO, J. C. M.; MORAES, L. C. Estimativa do surto de crescimento puberal pela avaliação das vértebras cervicais em radiografias cefalométricas laterais. Ortodontia, São Paulo, v. 34, n. 1, p. 51-60, jan./abr. 2001

3. BACCETTI, T.; FRANCHI, L.; MCNAMARA JUNIOR, J. A. An improved version of the cervical vertebral maturation (CVM) method for the assessment of mandibular growth. Angle Orthod, Appleton, v. 73, no. 4, p. 316-323, Aug. 2002.

4. BENEMANN, E.; SAMPAIO, R.; BERTHOLD, T. Idade biológica: indicadores do surto de crescimento. Ortodontia Gaúcha, Porto Alegre, v. 1, n. 1, p. 62-76, abr. 1997.

5. BJORK, A.; HELM, S. Prediction of the age of maximum puberal growth in body height. Angle Orthod, Appleton, v. 37, no. 2, p. 131-143, Apr. 1967.

6. BOWDEN, B. D. Epiphysal changes in the hand/wrist area as indicators of adolescent stage. Austr Orthod J, Sidney, v. 4, no. 3, p. 87-104, 1976.

7. CHAVES, A. P.; FERREIRA, R. I.; ARAÚJO, T. T. Maturação esquelética nas raças branca e negra. Ortodontia Gaúcha, Porto Alegre, v. 3, n. 1, p. 45-52, jan./jul. 1999.

8. COUTINHO, S.; BUSCHANG, P. H.; MIRANDA, F. Relationships between mandibular canine calcification stages and skeletal maturity. Am J Orthod Dentofacial Orthop, St. Louis, v. 140, no. 3, p. 262-268, Sept. 1993.

9. FISHMAN, L. S. Radiographic evaluation of skeletal maturation: a clinically oriented method based on hand-wrist film. Angle Orthod, Appleton, v. 52, no. 2, p. 88-112, Apr. 1982.

10. FISHMAN, L. S. Can cephalometric X-rays of the cervical columm be useds instead of hand-wrist X-rays to determine a patient's maturational age? Am J Orthod Dentofacial Orthop, St. Louis, v. 122, no. 1, p. 18A-19A, July 2002

11. FLORES-MIR, C. et al. Use of skeletal maturation based on hand-wrist radiographic analysis as a predictor of facial growth: a systematic review. Angle Orthod, Appleton, v. 74, no. 1, p. 118-124, Feb. 2004.

12. GARCÍA-FERNANDEZ $P$. et al. The cervical vertebrae as maturational indicators. J Clin Orthod, Boulder, v. 32, no. 4, p. 221-225, Apr. 1998.

13. GENEROSO, R. et al. Estudo da correlação entre idade cronológica e a maturação das vértebras cervicais em pacientes em fase de crescimento puberal. R Dental Press Ortodon Ortop Facial, Maringá, v. 8, n. 4, p. 19-36, jul./ago. 2003.

14. GRAVE, K. C.; BROWN. Skeletal ossification and the adolescent growth spurt. Am J Orthod, St. Louis, v. 69, no. 6, p. 611-619, June 1976
15. GUZZI, B. S. S.: CARVALHO L. S. Estudo da maturação óssea em pacientes jovens de ambos os sexos através de radiografias de mão e punho. Ortodontia, São Paulo, v. 3, n. 3, p. 49-58, set./dez. 2000

16. HASSEL, B.; FARMAN, A. G. Skeletal maturation evaluation using cervical vertebrae. Am J Orthod Dentofacial Orthop, St. Louis, v. 107, no. 1, p. 58-66, Jan. 1995.

17. KUCUKKELES, N. et al. Comparisons between cervical vertebrae and hand-wrist maturation for the assessment of skeletal maturity. J Clin Pediatr Dent, Birmingham, v. 24, no. 1, p. 47-52, 1999

18. LEITE, H. R.; O'REILLY, M. T.; CLOSE, M. J. Skeletal age assessment using first, second and third fingers of hand. Am J Orthod Dentofacial Orthop, St. Louis, v. 92, no. 6, p. 492-498, Dec. 1987.

19. MERCADANTE, M. M. N. Radiografia de mão e punho. In: VELLINI-FERREIRA, F. Ortodontia: diagnóstico e planejamento clínico. 4. ed. São Paulo: Artes Médicas, 2001. p. 189-216.

20. O' REILLY, M. T.; YANNIELLO, G. J. Mandibular growth changes and maturation of cervical vertebrae: a longitudinal cephalometric study. Angle Orthod, Appleton, v. 58, no. 2, p. 179-184, Apr. 1988.

21. SAN ROMÁN, P. et al. Skeletal maturation determined by cervical vertebrae development. Eur J Orthod, London, v. 24, no. 3, p. 303-341, 2002.

22. SANTOS, S. C. B. N. et al. Avaliação de um método de determinação do estágio de maturação esquelética utilizando as vértebras cervicais presentes nas telerradiografias em norma lateral. R Dent Press Ortodon Ortop Facial, Maringá, v. 3, n. 3, p. 67-77, maio/jun. 1998

23. SANTOS, S. C.; ALMEIDA, R. R. Estudo comparativo de dois métodos de avaliação da idade esquelética utilizando telerradiografias em norma lateral e radiografias carpais. Ortodontia São Paulo, v. 32, n. 2, maio/ago. 1999

24. SILVA FILHO, O. G.; SAMPAIO, L. L.; FREITAS, J. A. S. Avaliação de um método simplificado para estimar a maturação esquelética. Ortodontia, São Paulo, v. 25, n. 1, p. 21-36, jan./abr. 1992.

25. SIQUEIRA, V. C. V. et al. O emprego das radiografias da mão e do punho no diagnóstico ortodôntico. R Dental Press Ortodon Ortop Facial, Maringá, v. 4, n. 3, p. 20-29, maio/jun. 1999.

26. STRÖHER, D. V.; FREITAS, L.; TAVANO, O. A estimativa do surto de crescimento puberal, por meio do osso sesamóide e da menarca. Rev ABRO, Bauru, v. 5, n. 1, p. 42-46, jan./jul. 2004.

27. TAVANO, O.; ARMOND, M. C.; GENEROSO, R. Maturação das vértebras cervicais vistas através de radiografias cefalométricas laterais. Rev ABRO, Bauru, v. 1, n. 2, p. 15-24, maio/ ago. 2000

28. URSI, W. J. S. Determinação da maturidade esquelética através de radiografias carpais: sua importância na tratamento ortodôntico-ortopédico. In: INTERLANDI, S. Ortodontia: bases para a iniciação. 3. ed. Rio de Janeiro: Artes Médicas, 1994. p. 615.

29. UYSAL, T. et al. Relationship between dental and skeletal maturity in Turkish subjects. Angle Orthod, Appleton, v. 74, no. 5 , p. 657-664, Oct. 2004
Endereço para correspondência

Melissa Feres Damian

Faculdade de Odontologia - Universidade de Passo Fundo

Campus I - Bairro São José - BR 285- Km 171

CEP: 99.001-970 - Caixa Postal 611 - Passo Fundo/RS

E-mail: melissaodonto@upf.br 